\title{
Sleep-disordered breathing: Aids to diagnosis without a polysomnogram
}

\author{
A Peter, MB BCh, DA, FCP, Cert (Pulm), FCCP \\ Division of Pulmonology, Chris Hani Baragwanath Academic Hospital, Johannesburg, South Africa
}

Corresponding author: A Peter (alanpeter@ymail.com)

The South African population is suffering from an obesity epidemic. Sleep-disordered breathing (SDB), which includes obstructive sleep apnoea and obesity hypoventilation syndrome, is closely related to obesity. SDB may have serious health consequences if not asked about when taking a history related to sleep and sleep-deprivation symptoms. Unfortunately, a formal polysomnogram is available to very few patients who need the diagnosis confirmed. However, taking a sleep history, measuring the haemoglobin level and using a much smaller device in the comfort of a patient's bed can obviate the need for formal polysomnography.

Afr J Thoracic Crit Care Med 2018;24(4):139-142. DOI:10.7196/SARJ.2018.v24i4.223

Obesity has become a global epidemic, not only of developed countries but of Africa too. ${ }^{[1]}$ A previous demographic health survey among South Africans found that $29 \%$ of men and $56 \%$ of women are overweight or obese. ${ }^{[2]}$ Doctors seldom take a sleep history, because they think that a polysomnogram (PSG) is essential to diagnosing sleep-disordered breathing (SBD). The condition is therefore seldom picked up, and the consequences of neither obstructive sleep apnoea (OSA) nor obesity hypoventilation syndrome (OHS) are explained to patients. Added to this dilemma is the lack of resources to supply continuous positive airway pressure machines to the vast majority of the obese population, who can neither afford a sleep study nor pay for the machine. The present article aims to evaluate a simple questionnaire using information taken from a history and examination, haemoglobin ( $\mathrm{Hb}$ ) levels and the use of home sleeptesting with an ApneaLink device as an aid to diagnosing OSA. The metabolic consequences of SDB will also be outlined. With these simple tools, patient care and education can be enhanced to promote a greater resolve to lose weight.

\section{The polysomnogram: The current gold standard in diagnosing OSA}

The PSG is widely used as the gold standard in establishing SDB, and home sleep-testing devices are not as accurate. A formal PSG, however, is too expensive for most patients who need one, since the cost is between ZAR2 000 and ZAR5 000. It is a scarce resource for patients in the public sector, compared with those in the private sector. Mulgrew et al. ${ }^{[3]}$ demonstrated that combining the Epworth Sleepiness Scale (ESS), a sleep apnoea clinical score (measuring snoring, apnoea, neck circumference and blood pressure), and a home testing device measuring a respiratory disturbance index, showed a 0.94 positive predictive value $(94 \%$ accuracy) in making the diagnosis without a PSG. Not using a PSG results in a very small overdiagnosis of OSA; however, the benefits of a diagnosis or highly likely diagnosis, and therapy, outweigh the increased sensitivity. ${ }^{[3]}$

\section{Snoring - prevalence and health risks}

A large number of people snore at night. The Wisconsin Sleep Cohort Study involved 1843 women and 1670 men, and found that $28 \%$ of women and $44 \%$ of men were habitual snorers. Among the habitual snorers, $9 \%$ of women and $24 \%$ of men had OSA. ${ }^{[4]}$ Not all snorers have apnoea, but the vast of majority of OSA patients snore.

Snoring is three times more common in men than women, and the incidence increases in both sexes with ageing. ${ }^{[5]}$ The louder the snoring, the greater the risk of OSA developing. Mild snoring reaches between 40 and 50 decibels (db), moderate between 50 and $60 \mathrm{db}$ and severe above $60 \mathrm{db} \cdot{ }^{[6]}$ As a comparator, a whisper is in the region of $30 \mathrm{db}$, normal conversation is between 50 and $65 \mathrm{db}$ and a hair dryer and vacuum cleaner each operate at $70 \mathrm{db}$. Very loud snoring causes disturbed sleep for those who share the room at night.

Snoring without OSA is a risk for carotid artery atherosclerosis, while not affecting the femoral artery. ${ }^{[7]}$ Snoring on its own was not associated with an increased risk of hypertension. ${ }^{[8]}$

\section{The STOP-BANG questionnaire}

A questionnaire has been developed to detect OSA, since PSG is not widely available. A point is allocated to each of the following: snoring, tiredness, observed apnoea, pressure (hypertension), body mass index (BMI), age, neck circumference and gender (STOP-BANG; (Table 1) ${ }^{[9]}$ Standard values used in the STOP-BANG questionnaire are a BMI $>35 \mathrm{~kg} / \mathrm{m}^{2}$, age $>50$ years, neck circumference $>40 \mathrm{~cm}$ for females and $42.5 \mathrm{~cm}$ for males, and snoring loud enough to be heard through a closed door. A score of $\geq 3$ is considered a high risk for OSA, while $<3$ confers low risk.

The questionnaire shows high sensitivity, with scores of $>5$, to the maximum of 8 , identifying patients at risk for moderate to severe OSA, as confirmed by PSG. ${ }^{[10]} \mathrm{A}$ score $<2$ points excludes a risk of OSA. The specificity of this questionnaire, however, remains low, allowing many false positives, particularly for detecting mild sleep apnoea. Every hypertensive male $>50$ years old who snores immediately scores 3 in the questionnaire, and is seen as at risk of mild OSA. Harris et al.. ${ }^{[1]]}$ 
in a study that used PSG, found that of 110 obese patients showing moderate OSA, a STOP-BANG questionnaire score of $\geq 4$ showed a sensitivity of $85 \%$, but a specificity of $44 \%$. A low specificity makes the test an unreliable diagnostic tool; however, the high sensitivity confers good screening ability.

\section{The Epworth Sleepiness Scale}

Being tired is a subjective feeling. To better answer the second question in the STOP-BANG questionnaire regarding tiredness, the Epworth Sleepiness Scale (ESS; Table 2) ${ }^{[12]}$ attempts to alert both clinician and patient to a degree of tiredness that may well relate to SDB robbing one of restorative sleep.

\section{Improving the poor specificity of the STOP-BANG questionnaire}

\section{ApneaLink device}

If a STOP-BANG questionnaire that has a score of $\geq 4$ is coupled with readings from an ApneaLink device (available in South Africa), which measures pulse oximetry, pulse, respiration, apnoeas and hypopnea, then the specificity of the STOP-BANG score is improved. Using an Apnoea Hypopnoea Index (AHI) score of $\geq 15$ raised the specificity from $44 \%$ to $89 \%$; if hypertension was present, it rose to $89 \%$; if the neck circumference was $>40 \mathrm{~cm}$, it was $92 \%$, and for males $93 \%$. High sensitivities are obtained in all three instances. ${ }^{[1]}$ Compared with PSG, ApneaLink recordings have $100 \%$ specificity, but $54 \%$ sensitivity, if using an AHI of $15 .^{[15]}$ The high specificity augments the STOP-BANG score. The ApneaLink device is small, and does not require an hour to

\begin{tabular}{l} 
Table 1. STOP-BANG questionnaire (1 point per yes answer) \\
\hline Sign or symptom \\
\hline Snoring heard through closed door, louder than \\
talking \\
Tiredness, fatigue, sleepy during the day \\
Observed apnoea \\
Pressure (hypertension) \\
Body mass index $>35 \mathrm{~kg} / \mathrm{m}^{2}$ \\
Age $>50$ years \\
Neck circumference $>40 \mathrm{~cm}$ in females, \\
$>42.5 \mathrm{~cm}$ males \\
Male sex
\end{tabular}

set up, as does a formal PSG, and it can be used easily at home (Fig. 1). Nasal cannulae are inserted, and the small device is strapped to the chest wall, with a pulse oximeter probe on the finger (Fig. 2). The current price for all components, as well as the software programme that analyses the sleep study, is ZAR14 090, discounted for state institutions, while private purchase is around ZAR17 000 . The only consumable is the nasal cannulae tubing. Using the device multiple times on inpatients in state hospitals, or provided to private patients, is a more economical sleep study method than using the formal PSG in a sleep laboratory.

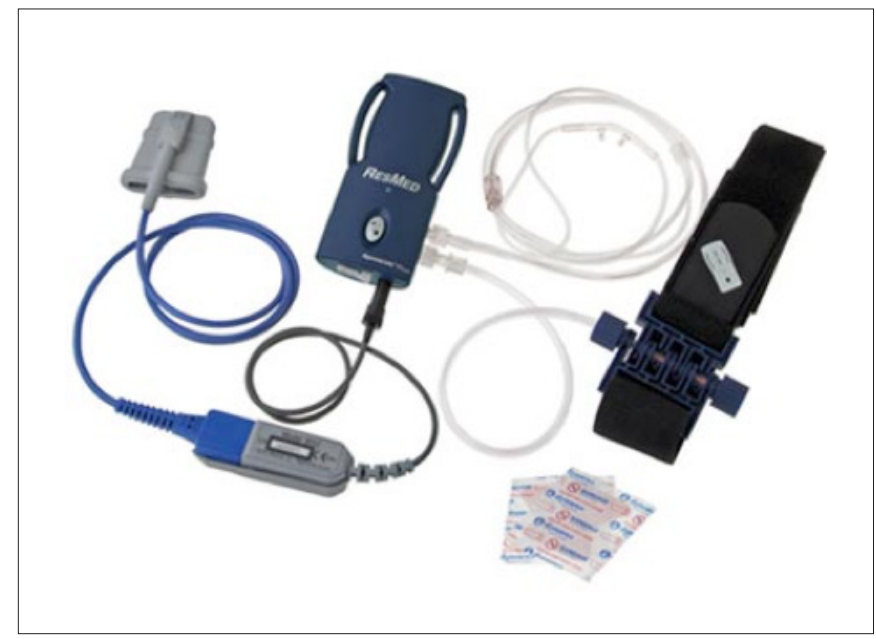

Fig 1. ApneaLink device.

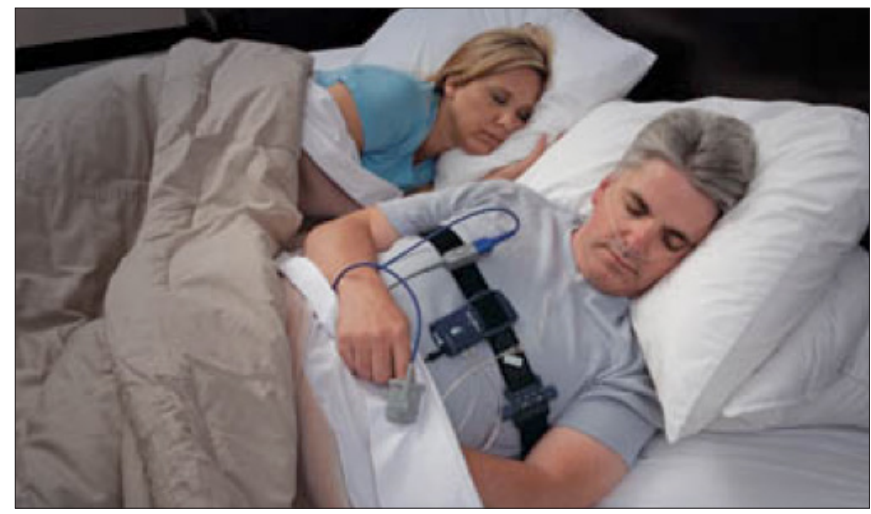

Fig 2. ApneaLink Plus applied to the patient.

Table 2. Epworth Sleepiness Scale (ESS)*

\begin{tabular}{|c|c|c|c|c|}
\hline \multirow[b]{2}{*}{ Situation } & \multicolumn{4}{|c|}{ Likelihood of dozing (point score) } \\
\hline & Never (0) & Slight (1) & Moderate (2) & High (3) \\
\hline \multicolumn{5}{|c|}{ Sitting reading } \\
\hline \multicolumn{5}{|c|}{ Watching television } \\
\hline \multicolumn{5}{|c|}{ Sitting inactive in a public space } \\
\hline \multicolumn{5}{|c|}{ Passenger in a car for 1 hour } \\
\hline \multicolumn{5}{|c|}{ Lying down in the afternoon } \\
\hline \multicolumn{5}{|c|}{ Sitting talking to someone } \\
\hline \multicolumn{5}{|c|}{ Sitting quietly after lunch (no alcohol) } \\
\hline \multicolumn{5}{|c|}{ Sitting in a car stopped for a few minutes in traffic } \\
\hline \multicolumn{5}{|c|}{$\begin{array}{l}\text { *A score of up to } 6 \text { points indicates that enough sleep is being obtained }\left(4-8 \text { points }=\text { normal). A survey report }{ }^{[13]} \text { found that }>30 \% \text { of working people in the USA sleep }<6 \text { hours. African ethnicity is associated }\right. \\
\text { with higher sleep deprivation, as is night-shift work. A score between } 9 \text { and } 15 \text { points signals the need to seek medical advice, and } \geq 16 \text { points represents a dangerous risk to health. ESS alone is not sufficient } \\
\text { to make a diagnosis of OSA.14] }\end{array}$} \\
\hline
\end{tabular}




\section{Haemoglobin levels}

Chung et al. ${ }^{[16]}$ analysed 383 patients with proven OSA, and evaluated the use of a $\mathrm{Hb}$ level. They found that $\mathrm{Hb}$ levels of $\geq 16 \mathrm{~g} / \mathrm{dL}$ for men, and $\geq 15 \mathrm{~g} / \mathrm{dL}$ for women, when added to a STOP-BANG questionnaire score of $\geq 3$ points, improved the specificity from $35 \%$ to $94 \%$ for all sleep apnoea, and from $24 \%$ to $80 \%$ for severe OSA.

Using either the ApneaLink device or an $\mathrm{Hb}$ level improves the poor specificity levels of STOP-BANG questionnaire scores between 3 and 5 . A STOP-BANG score $\geq 6$ points on its own showed $82 \%$ specificity for OSA as measured by PSG.

\section{SDB in the non-obese}

Non-obese individuals may also have SDB, but without the doctor being alerted by obesity, and therefore not taking a patient's history of sleep, the non-obese sleepdisordered patient can be missed. In a study by Cadavid, ${ }^{[17]} 17 \%$ of 611 patients referred for OSA assessment were non-obese, and half of these non-obese patients proved to have OSA. The greatest predictors of OSA in the non-obese person proved to be age and gender, rather than sleepiness and ethnic origin. A 1-point increase in BMI signaled an $8 \%$ higher odds risk, while being male increased the odds 11.7 fold and a 10-year age increase resulted in a $44 \%$ increase in the odds of OSA in the non-obese patient.

\section{Health risks of OSA}

The pathophysiology of SDB described in Fig. 3 is adapted from an article by Bradley and Floras. ${ }^{[18]}$ The two main drivers are hypoxia and increased negative intrathoracic pressures generated when breathing against an obstructed airway. Continuous positive airway pressure (CPAP) both addresses hypoxia and, by keeping the airway open, decreases the raised negative intrathoracic pressures by opening the airway and releasing the negative intrathoracic pressure so that the lung is able to expand with incoming air. The sympathetic pathway and inflammatory cytokine generation, both affecting the endothelium and causing inflammation, together with increased venous return to the right side of the heart, results in an increased risk of hypertension, cardiac failure, atheroma,

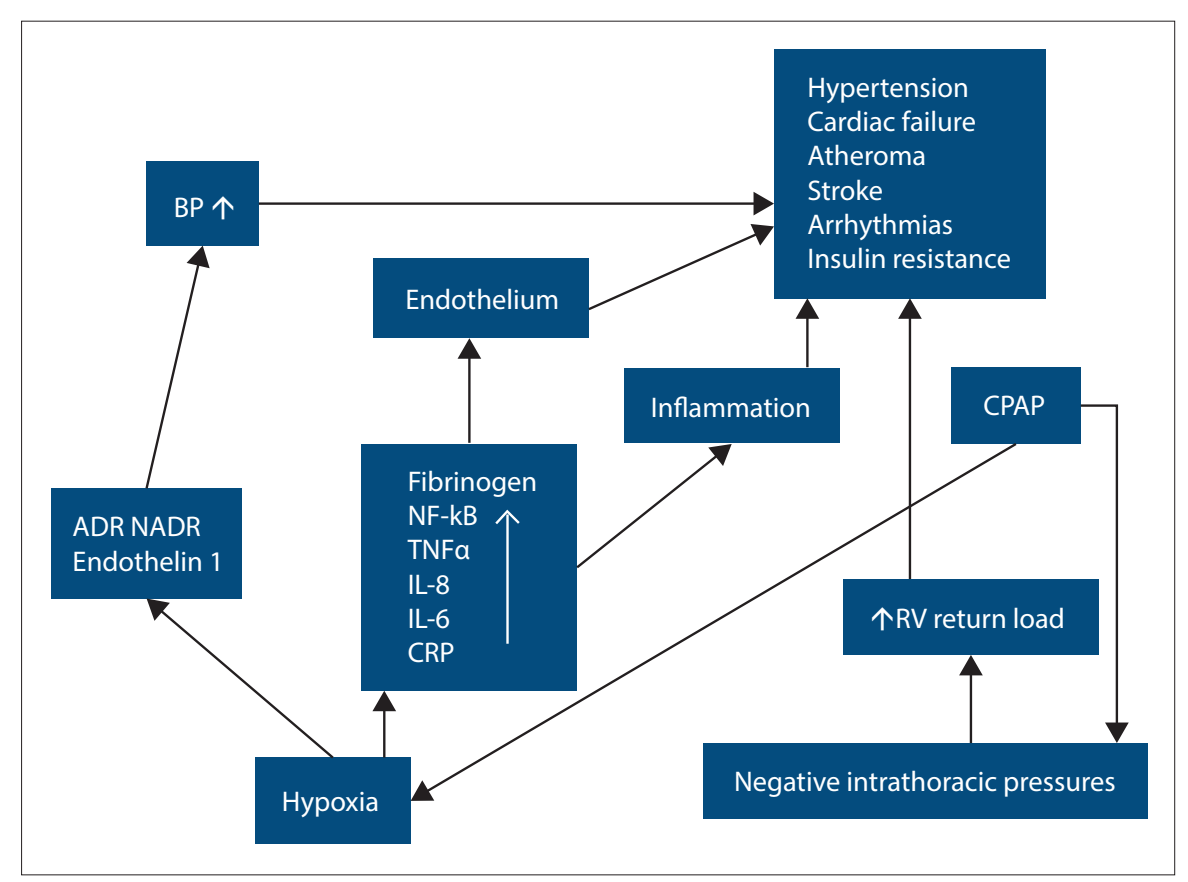

Fig. 3. Metabolic consequences of sleep-disordered breathing and the role of continuous positive airway pressure $(C P A P) .(B P=$ blood pressure; $(N) A D R=($ nanoparticle $)$ adriamycin; $N f-k B=$ nuclear factor kappa-light-chain-enhancer of activated B cells; TNF = tumour necrosis factor; $I L=$ interleukin; $C R P=C$-reactive protein; $R V=$ right ventricular.)

stroke arrhythmias and insulin resistance.

The Sleep Heart Health Study ${ }^{[19]}$ established an association between OSA and diabetes. Pallayova et al. ${ }^{[20]}$ demonstrated that in normal glucose metabolism, patients with severe obesity and moderate to severe OSA, decreased levels of pancreatic beta-cell functioning, together with increased insulin resistance, were found. Both are causal in the development of diabetes. Raised tumour necrosis factor $\alpha$ (TNFa) and cytokine interleukin -6 (IL-6) levels correlated with OSA-related oxyhaemoglobin desaturations.

It is controversial whether CPAP can mitigate the development or acceleration of diabetes, and evidence suggests that it may not. ${ }^{[20]}$

\section{The difference between OSA and OHS}

Characteristic findings in OHS are a raised serum bicarbonate level, and a raised $\mathrm{CO}_{2}$ of $\geq 45 \mathrm{mmHg}$ in an arterial blood gas analysis, which are absent in pure OSA. Pulmonary hypertension exists in $15 \%$ of OSA alone, while the incidence is between $60 \%$ and $88 \%$ in OHS, and similarly, the risk of intensive care unit (ICU) admission in OSA alone is $6 \%$, but $40 \%$ in OHS. ${ }^{[21]}$ Mortality for patients with OSA admitted to hospital is $9 \%$, compared with 23\% for those with OHS. ${ }^{[21]} \mathrm{OHS}$ therefore confers greater health risks, and signals a dire need for weight reduction. Ten to $20 \%$ of the OSA population will have OHS. African and Asian ethnicity confers a higher risk of OHS, owing to cephalometric differences in cranial structure, and consequent airway diameters, in addition to obesity. ${ }^{[21]}$

\section{Leptin}

Why only a third of very obese patients develop OHS is not clear. ${ }^{[22]}$ Leptin is a hormone produced on chromosome 7 by adipocytes. ${ }^{[23]}$ Leptin suppresses appetite, increases energy expenditure and stimulates the respiratory centre. Leptin resistance in obese people leads to increased food intake, and the inability to increase tidal volume and respiratory rate to cope with the increased work of breathing associated with obesity. Hypercapnia in OHS is postulated to be a cause of insufficient drive on the respiratory centre caused by leptin resistance, or low leptin levels. Leptin resistance is an acquired defect. Leptin acts on the hypothalamus to initiate satiety. Sustained overeating causes high levels of leptin in the cerebrospinal fluid, which leads to the development of hypothalamic receptor resistance. The hypothalamic receptors then fail to initiate 
satiety. Leptin resistance may also arise from mutations, causing resistance in downstream leptin regulator proteins. ${ }^{[24]}$

\section{Conclusion}

So what can we offer a patient if a PSG is not feasible or available? We should take a sleep history, and look for symptoms of sleep deprivation, either using the STOP-BANG questionnaire or the ESS. Measuring a $\mathrm{Hb}$ and $\mathrm{PaCO}_{2}$ in patients who have a $\mathrm{BMI}>30 \mathrm{~kg} / \mathrm{m}^{2}$ provides clues to the metabolic consequences of hypoxia and OHS (the raised $\mathrm{PaCO}_{2}$ ). Using a small device to record an $\mathrm{AHI}$ index in the comfort of a patient's home or hospital bed further establishes a diagnosis of SDB. An electrocardiograph that shows signs of pulmonary hypertension when SDB is present, and other causes have been excluded, is more likely in OHS than OSA, the former of which carries a worse prognosis. Since other illnesses (hypertension, diabetes, cardiovascular disease) are associated with SDB, education about the much wider implications of merely snoring may motivate patients to lose weight.

Acknowledgements. None.

Author contributions. Sole author.

Funding. None.

Conflicts of interest. None.

1. Goedecke JH, Jennings CL, Lambert EV. Obesity in South Africa. In: Steyn K, Fourie J, Temple N, eds. Chronic Diseases of Lifestyle in South Africa: 1995 - 2005. Technical Report. Cape Town: South African Medical Research Council, 2006.

2. Puoane T, Steyn K, Bradshaw, et al. Obesity in South Africa: The South African Demographic and Health Survey. Cape Town: Medical Research Unit Tygerberg Hospital, 2002:1038-1048.

3. Mulgrew AT, Fox N, Ayas NT, Ryan CF. Diagnosis and initial management of obstructive sleep apnea without polysomnography: A randomized validation study. Ann Int Med 2007;146(3):157-166.

4. Young T, Palta M, Dempsey J, Skatrud J, Weber S, Badr S. The occurence of sleep disordered breathing among middle aged adults. N Eng J Med 1993;328(17):12301235. https://doi.org/10.1056/NEJM199304293281704

5. Ng TP, Seow A, Tan WC. Prevalence of snoring and sleep breathing-related disorders in Chinese, Malay and Indian adults in Singapore. Eur Respir J 1998;12(1):198-203

6. Maimon N, PJ Hanly. Does snoring intensity correlate with the severity of obstructive sleep apnea?. J Clin Sleep Med 2010;6(5):475-478.

7. Lee SA, Amis T, Byth K, et al. Heavy snoring as a cause of carotid artery atherosclerosis. Sleep 2008;31(9):1207-1213.
8. Hoffstein V. Blood pressure, snoring, obesity, and nocturnal hypoxaemia. Lancet 1994;344(8923):643-645.

9. Chung F, Yegneswaran B, Liao P, et al. STOP questionnaire: A tool to screen patients for obstructive sleep apnea. Anesthesiology 2008;108(5):812-821. https://doi. org/10.1097/ALN.0b013e31816d83e4

10. Chung F, Subramanyam R, Liao P, Sasaki E, Shapiro C, Sun Y. High STOP-Bang score indicates a high probability of obstructive sleep apnoea. Br J Anaest 2012;108(5):768775. https://doi.org/10.1093/bja/aes022

11. Harris D, Sokolow B, Sundar K, Ludwig K. Comparative study of the stop-bang questionnaire, Apnealink, and polysomnography for obstructive sleep apnea. Chest 2011;140(4):944A. https://doi.org/10.1378/chest.1118750

12. Johns MW. A new method for measuring daytime sleepiness: The Epworth Sleepiness Scale. Sleep 1991;14(6):540-545.

13. Luckhaupt SE. Short sleep duration among workers - United States, 2010. National Health Interview Survey Morbidity and Mortality Weekly Report 2012;61:281-285.

14. Mondal P, Gjevre JA, Taylor-Gvejre RM, Lim HJ. Do Pittsburgh Sleep Quality Index scores and Epworth Sleepiness Scale scores correlate in a patient population referred for polysomnography? Chest 2012;142(4 Suppl):1063A. https://doi.org/10.1378/ chest. 1389225

15. Deeb A, Abou Joude S, Winkle M. Is the Apnea Link a reliable tool in the diagnosis of obstructive sleep apnea (OSA)? Chest 2012;142(4 Suppl):1010A. https://doi. org/10.1378/chest.1388736

16. Chung F, Liao P, Yang Y, Elsaid H, Islam S. Combining hemoglobin level with STOP-Bang questionnaire improved specificity to detect OSA. Chest 2011;140(4 Suppl):819A. https://doi.org/10.1378/chest.1118443

17. Cadavid JC. Clinical predictors of sleep apnoea in non-obese individuals: Are we heading towards universal screening? Chest 2009;136(4):59S. https://doi.org/10.1378/ chest.136.4_meetingabstracts.59s-g

18. Bradley AT, Floras JS. Obstructive sleep apnoea and its cardiovascular consequences. Lancet 2009;373(9657):82-93. https://doi.org/10.1016/S0140-6736(08)61622-0

19. Punjabi NM, Shahar E, Redline S, et al. Sleep-disordered breathing, glucose intolerance and insulin resistance: The Sleep Heart Health Study. Am J Epidemiol 2004;160(6):521-530. https://doi.org/10.1093/aje/kwh261

20. Pallayova M, Steele K, Magnuson TH, et al. Sleep apnoea predicts distinct alterations in glucose homeostasis and biomarkers in obese adults with normal and impaired glucose metabolism. Cardiovasc Diabetol 2010;9:83-91. https://doi.org/10.1186/14752840-9-83

21. Mokhlesi B. Obesity hypoventilation syndrome. Chest 2007;132(4):1322-1336 https://doi.org/10.1378/chest.07-0027

22. Nowbar S, Burkart KM, Gonzales R, et al. Obesity-associated hypoventilation in hospitalised patients: Prevalence, effects, and outcome. Am J Med 2004;116(1):1-7.

23. Isse $\mathrm{N}$, Ogawa $\mathrm{Y}$, Tamura $\mathrm{N}$, et al. Structural organisation and chromosomal assignment of the human obese gene. J Biol Chem 1995;270(46):27728-27733.

24. Klok D, Jakobsdottir S, Drent ML. The role of leptin and ghrelin in the regulation of food intake and body weight in humans: A review. Obes Rev 2007;8(1):21-34. https:// doi.org/10.1111/j.1467-789X.2006.00270.x

Accepted 29 November 2018. 